\title{
Abcès de la prostate
}

\author{
Reza Pishdad MD, Sean Sullivan DO, Oranus Mohammadi MD
}

- Citation : CMAJ 2021 February 22;193:E290. doi : 10.1503/cmaj.200470-f

Voir la version anglaise de l'article ici : www.cmaj.ca/lookup/doi/10.1503/cmaj.200470

$\mathbf{U}$

n homme de 72 ans s'est présenté au service des urgences en raison d'une fièvre, d'une dysurie et de douleurs rectales, ainsi que de mictions fréquentes et impérieuses, symptômes qui persistaient depuis 3 semaines. II avait depuis 10 ans un diagnostic de diabète de type 2 et d'hypertrophie bénigne de la prostate, traités respectivement par la prise de metformine et de finastéride. L'examen de la prostate par toucher rectal a révélé une masse palpable douloureuse et fluctuante. Il avait une numération leucocytaire de $13,5 \times 10^{9} / \mathrm{L}$ (plage normale 4-10 $\times 10^{9} / \mathrm{L}$ ) et un taux de créatinine sérique de $141 \mu \mathrm{mol} / \mathrm{L}$ (valeur de référence $114 \mu \mathrm{mol} / \mathrm{L}$ ). L'analyse microscopique de l'urine a fait état d'une bactériurie modérée; des tests par bandelettes ont donné un résultat fortement positif pour la leucocyte estérase et positif pour les nitrites. Après l'établissement d'un diagnostic de prostatite, le patient a été hospitalisé, et on a entamé une antibiothérapie intraveineuse par ceftriaxone $(1 \mathrm{~g} / \mathrm{j})$.

Comme le patient était encore fébrile plus de 48 heures après le début du traitement, nous avons effectué une tomodensitométrie abdominopelvienne avec agent de contraste, qui a montré une atténuation hétérogène de la prostate, laissant soupçonner un abcès de la prostate (figure 1 ; annexe 1 , accessible en anglais au www.cmaj.ca/lookup/doi/10.1503/cmaj.200470/tab -related-content). Une culture urinaire a permis d'identifier Klebsiella pneumoniae; l'hémoculture était négative. Quelques jours après l'hospitalisation, une sonde à demeure a été posée en raison de difficultés à uriner. Le patient a finalement subi une résection transurétrale de la prostate avec drainage de l'abcès. L'analyse histopathologique d'un échantillon de biopsie a montré une inflammation accompagnée de nécrose ainsi que la formation d'un abcès. Le patient ayant développé de la rétention urinaire après la résection, nous lui avons prescrit de la ciprofloxacine administrée par voie orale, puis nous l'avons renvoyé à la maison avec la sonde à demeure, qui a été retirée après 2 jours. Il ne s'est plaint d'aucun symptôme à sa rencontre de suivi après 6 semaines.

Les abcès de la prostate surviennent chez $2,7 \%$ des cas de prostatite bactérienne aiguë ${ }^{1}$, une affection qui touche surtout les patients âgés atteints d'un diabète sucré. Le cathétérisme à long terme, la manipulation de la prostate, l'obstruction urétrale et l'immunodéficience sont aussi des facteurs prédisposants ${ }^{1}$. Les bacilles Gram négatif et les entérocoques sont le

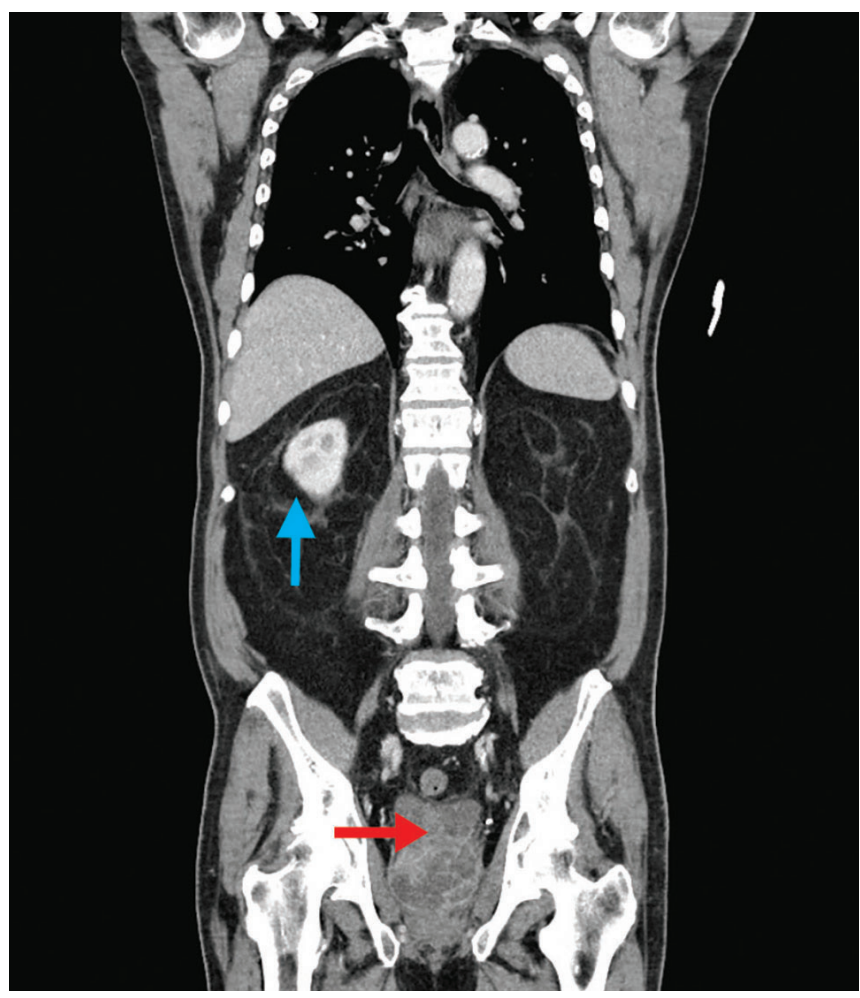

Figure 1 : Tomodensitométrie abdominopelvienne (plan coronal) chez un homme fébrile de 72 ans se plaignant de douleurs rectales. On y voit des stries apparentes dans l'espace périrénal droit (flèche bleue). Hypertrophie $(7 \mathrm{~cm}$ de diamètre) et atténuation hétérogène de la prostate avec accumulations de densité liquidienne non marquées laissant soupçonner un abcès de la prostate (flèche rouge).

plus souvent en cause $\mathrm{e}^{1}$. L'abcès de la prostate et la prostatite bactérienne ont des symptômes similaires ${ }^{1}$, mais une prostate fluctuante au toucher rectal peut être un signe d'abcès sousjacent, tout comme l'observation, à la TDM, d'une hypertrophie et d'une atténuation hétérogène de la prostate avec accumulations de densité liquidienne non marquées. La prise en charge

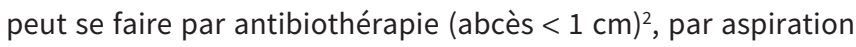
échoguidée transrectale, par résection transurétrale de la prostate ou par drainage à ciel ouvert ${ }^{3,4}$. L'aspiration échoguidée transrectale est la méthode à privilégier en raison de son faible risque de complications et du fait qu'elle peut être répétée. Cependant, le risque d'échec est élevé pour les abcès ayant un 
diamètre supérieur à $3 \mathrm{~cm}$ ou un aspect anéchogène ou hétérogène; pour ce type de lésion, la résection transurétrale de la prostate est préférable ${ }^{4}$. L'âge avancé, une forte fièvre, la rétention urinaire, l'hémoculture positive et l'hyperplasie bénigne de la prostate sont associés à un pronostic sombre ${ }^{1}$.

\section{Références}

1. Coker TJ, Dierfeldt DM. Acute bacterial prostatitis: diagnosis and management. Am Fam Physician 2016;93:114-20.

2. Chou YH, Tiu CM, Liu JY, et al. Prostatic abscess: transrectal color Doppler ultrasonic diagnosis and minimally invasive therapeutic management. Ultrasound Med Biol 2004;30:719-24.

3. Basiri A, Javaherforooshzadeh A. Percutaneous drainage for treatment of prostate abscess. Urol J 2010;7:278-80.

4. Abdelmoteleb H, Rashed F, Hawary A. Management of prostate abscess in the absence of guidelines. Int Braz J Urol 2017;43:835-40.

Intérêts concurrents : Aucun déclaré.

Cet article a été révisé par des pairs.

Les auteurs ont obtenu le consentement du patient.

Affiliations : Département de médecine interne (Pishdad, Sullivan), Rutgers New Jersey Medical School, Newark, N.J.; Département de médecine interne (Mohammadi), Aventura Hospital and Medical Center, Miami, Fla.

Propriété intellectuelle du contenu : Il s'agit d'un article en libre accès distribué conformément aux modalités de la licence Creative Commons Attributions (CC BY-NC-ND 4.0), qui permet l'utilisation, la diffusion et la reproduction dans tout médium à la condition que la publication originale soit adéquatement citée, que l'utilisation se fasse à des fins non commerciales (c.-à-d. recherche ou formation) et qu'aucune modification ni adaptation n'y soit apportée. Voir : https://creativecommons.org/licenses/by-nc-nd/4.0/deed.fr.

Correspondance : Reza Pishdad, reza_pishdad@yahoo.com

Les images cliniques sont choisies pour leur caractère particulièrement intéressant, classique ou impressionnant. Toute soumission d'image de haute résolution claire et bien identifiée doit être accompagnée d'une légende aux fins de publication. On demande aussi une brève explication (300 mots maximum) de la portée éducative des images, et des références minimales. Le consentement écrit du patient au regard de la publication doit être obtenu avant la soumission. 